\title{
Topografia do cone medular do sauim (Saguinus midas)
}

\author{
Topography of the medullary cone in sauim (Saguinus midas)
}

\author{
Danielli Martinelli Martins ${ }^{\mathrm{I}}$ Luane Lopes Pinheiro ${ }^{\mathrm{I}}$ Ana Rita Lima' ${ }^{\mathrm{I}}$ Luiza Corrêa Pereira ${ }^{\mathrm{II}}$ \\ Érika Renata Branco ${ }^{I^{*}}$
}

\section{RESUMO}

Primata endêmico do Brasil, especificamente da floresta Amazonica, o Sauim alimentam-se de pequenos vertebrados, invertebrados, exudatos, flores, néctar, folhas e fungos, cuja morfologia é pouco conhecida. Assim, objetivouse estabelecer parâmetros morfométricos e topográficos do cone medular dessa espécie, que sirvam de base para a prática das anestesias epidurais. Para tanto, foram avaliados quatro exemplares, provenientes da Universidade Federal Rural da Amazônia (UFRA). O Saguinus midas apresentou sete vértebras lombares e três vértebras sacrais. Após a dissecação do cone medular, registrou-se os aspectos anatômicos de interesse, enfatizando seu início (base) e seu término (ápice), que, após mensuração, revelou base em nível de L4 e ápice em $S 2$, com comprimento médio de $5,38 \mathrm{~cm}$, o que leva a sugerir a região lombosacral como sendo a mais adequada para o desenvolvimento da prática de anestesias epidurais.

Palavras-chave: cone medular, sauim, topografia, Saguinus midas.

\section{ABSTRACT}

Primate endemic to Brazil, specifically in the Amazon rainforest, the Sauim feed on small vertebrates, invertebrates, exudates, flowers, nectar, leaves and fungi whose morphology is poorly known. Thus, the objective was to establish morphometric parameters and topographical conus of this species, as a basis for the practice of epidural anesthesia. For this purpose, four samples were evaluated, from the Universidade Federal Rural da Amazonia (UFRA). The Saguinus midas had seven lumbar vertebrae and three sacral vertebrae. After dissection of the conus, we recorded the anatomy of interest, emphasizing its beginning (base) and its end (apex), revealed that after measuring the level of base peak in L4 and S2, with an average length of $5.38 \mathrm{~cm}$, which leads us to suggest the lumbosacral region as the most suitable for the development of the practice of epidural anesthesia.

Key words: medullary cone, sauim, topography, Saguinus midas.

\section{INTRODUÇÃO}

O Sauim (Saguinus midas) é um mamífero pertencente à família Callitrichidae e ao gênero Saguinus. Possui a pele da face e pêlos totalmente negros, assim como cabeça e ombros, sendo o restante do corpo e pernas mosqueados de preto e amarelo ou de preto e vermelho. Mãos e pés também negros (ARAÚJO et al., 2000). Essa espécie ocorre na Guiana Inglesa, Suriname, Guiana Francesa e no Brasil, mais especificadamente ao norte do Rio Amazonas e leste do Rio Negro, em direção à costa do Atlântico e ao sul do Rio Amazonas, entre os rios Xingu e Gurupi, incluindo a Ilha do Marajó (HERSHKOVITZ, 1977).O conhecimento da morfologia do sistema nervoso das diversas espécies animais assume considerável importância na clínica médico-cirúrgica veterinária, tendo em vista que a anestesia epidural, obtida por injeção de anestésicos locais no espaço epidural, que é o espaço compreendido entre a dura-máter e o canal vertebral, é uma técnica de anestesia regional muito utilizada por oferecer boa margem de segurança (HOPKINS, 1935; HILBERY, 1992; CARVALHO et al., 2003; DYCE et al., 2004). Para tanto, o conhecimento da topografia do cone medular tem sua importância na informação quanto ao local mais indicado para aplicação dessa prática anestésica sem resultar em lesões, pois o espaço epidural varia conforme a espécie animal, de acordo com o local do término

ILaboratório de Pesquisa Morfológica Animal (LaPMA), Faculdade de Medicina Veterinária, Universidade Federal Rural da Amazônia (UFRA), Avenida Presidente Tancredo Neves, 2501, 66077-530, Belém, PA, Brasil. E-mail: erika.branco@ufra.edu.br. *Autor para correspondência.

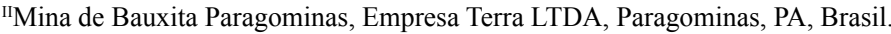


da medula espinhal (HILBERY, 1992; FANTONI \& CORTOPASSI, 2002; DYCE et al., 2004), além de servir como referência para punção de líquor para exames laboratoriais e ainda facilitar a localização de lesões nervosas centrais em níveis vertebrais específicos (DYCE et al., 2004).

A topografia do cone medular pode variar de acordo com a espécie e a idade do animal, ocorrendo entre as últimas vértebras lombares e as primeiras sacrais (RAO, 1990; RAO et al., 1993; DYCE et al., 2004).

Diante desse cenário e da escassez literária desse tema em primatas não-humanos, este estudo visionou determinar a topografia do cone medular do $\boldsymbol{S}$. midas, a fim de subsidiar conhecimento para futuras abordagens de práticas anestésicas epidurais.

\section{MATERIAL E MÉTODOS}

Foram utilizados quatro exemplares de Saguinus midas, fêmeas, adultas, provenientes da Universidade Federal Rural da Amazônia (UFRA), sob autorização no 485/2009, 522/2009, que foram a óbito por atropelamento.

Para a fixação e conservação do animal, foi utilizada solução aquosa de formol a $10 \%$ mediante aplicações intramusculares, subcutâneas e intracavitárias, e, em seguida, os animais foram mantidos submersos na mesma solução por um período mínimo de sete dias, em recipiente adequado.
As dissecações foram feitas por meio de incisão longitudinal da pele na linha mediana dorsal, desde a região torácica até a base da cauda, seguido de rebatimento da pele, promoveu-se a remoção da musculatura epiaxial, resultando na exposição da coluna vertebral. Posteriormente foram retirados os arcos vertebrais, expondo-se a medula espinhal envolta pelas meninges, permitindo a localização do cone medular, bem como sua relação topográfica com as vértebras. Em seguida, realizou-se a mensuração do comprimento do cone medular, utilizando-se paquímetro digital Dc-6 Western. Toda nomenclatura adotada foi baseada na INTERNATIONAL COMMITTEE ON VETERINARY GROSS ANATOMICAL NOMENCLATURE (2005).

\section{RESULTADOS E DISCUSSÃO}

Os exemplares de sauim apresentaram sete vértebras lombares e três vértebras sacrais (Figura 1A), sendo que, em todos os exemplares, a base do cone medular, disposta caudalmente a intumescência lombar, localizou-se ao nível da 4a vértebra lombar (L4), enquanto que e o ápice ao nível da $2^{\text {a }}$ vértebra sacral (S2), sendo seguido pela cauda equina (Figura 1B). Quando mensurado, o cone medular revelou média de $5,14 \mathrm{~cm}$ de comprimento a partir da variação apresentada entre os animais (Tabela 1).

Ainda que pertencendo a mesma família (Callitrichidae), ao contrário dos achados no presente estudo, MACHADO et al. (1992) registraram

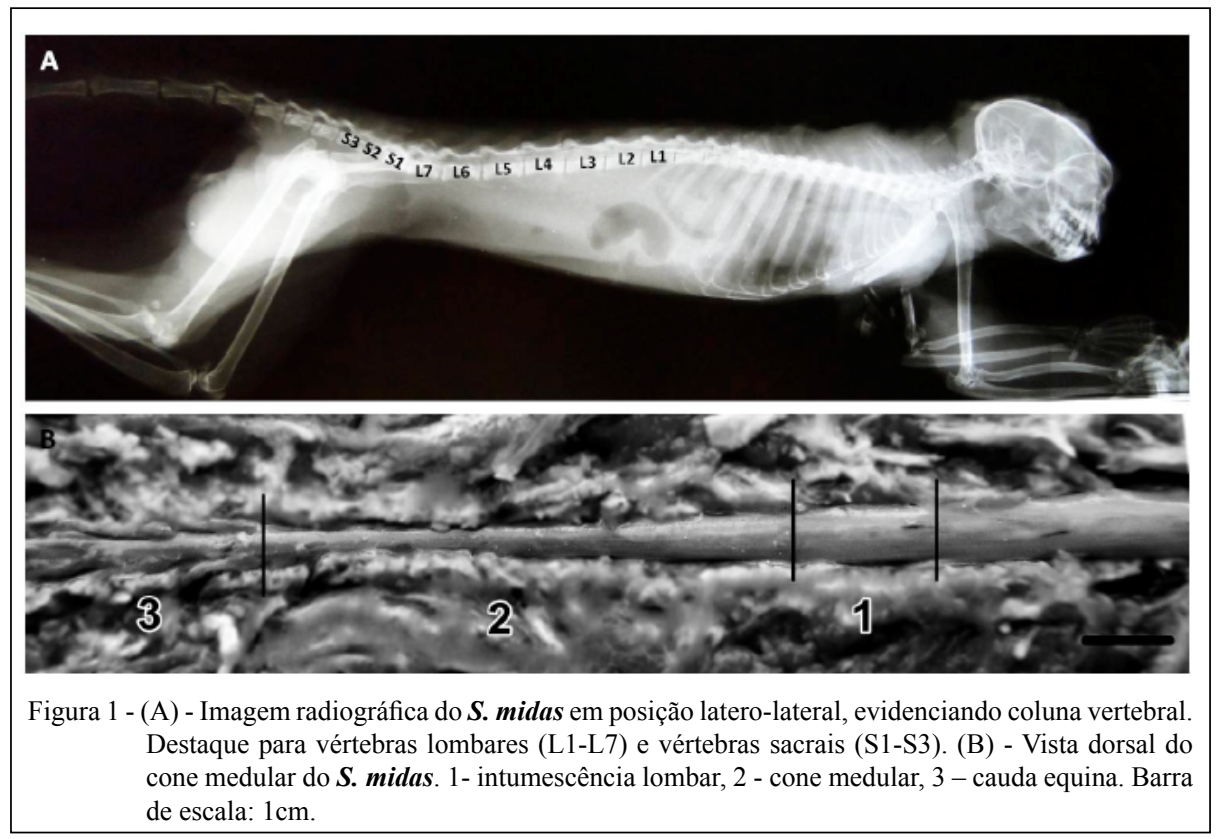

Ciência Rural, v.43, n.6, jun, 2013. 
Tabela 1 - Representação da topografia do cone medular, base e ápice, e seu comprimento $(\mathrm{cm})$, em quatro exemplares de Sauim (S. midas).

\begin{tabular}{lccc}
\hline No animal & Base do cone & Ápice do cone & $\begin{array}{c}\text { Comprimento } \\
\text { do cone }\end{array}$ \\
\hline 01 & L4 & S2 & 5,61 \\
02 & L4 & S2 & 4,65 \\
03 & L4 & S2 & 5,9 \\
04 & L4 & S2 & 4,4 \\
\hline
\end{tabular}

topografia diferente do cone medular do sagui-detufo-branco (Callithrix jacchus), estando disposto entre L2 e L4, enquanto que no Sauim (S. midas) a mesma estrutura dispôs-se entre L4 e S2.

Quando comparado ao grupo dos carnívoros, o Sauim apresenta a topografia do cone medular bastante semelhante ao da jaguatirica (Leopardus pardalis), a qual foi registrada por BRÍGIDA et al. (2010) localizando-se entre L4-S3, porém, com uma mensuração bastante diferente, $8,5 \mathrm{~cm}$ contra os $5,14 \mathrm{~cm}$ registrados no $\boldsymbol{S}$. midas.

Já para o quati (Nasua nasua) (GREGORES et al., 2010) e para o gato mourisco (Herpailurus yagouaroundi) (CARVALHO et al., 2003), não só a topografia quanto o comprimento apresentam certa similaridade com os achados no Sauim, registrando-se o cone medular no quati entre L5-6 e S3 com 5,2-5,8cm e no gato mourisco estando entre L6 e $\mathrm{S} 2$ com $5 \mathrm{~cm}$ de comprimento.

Ainda dentro da categoria dos carnívoros terrestres, encontra-se o lobo guará (Chrysocyon brachyurus), que, de acordo com MACHADO et al. (2002), revelou não apenas uma topografia relativamente diferente das supra mencionadas, principalmente quando comparado com o sauim, localizando-se entre L3 e L5-6, bem como o comprimento mais expressivo $(6,5 \mathrm{~cm})$.

Confrontando os achados anteriores com carnívoros de vida aquática, a ariranha (Pteronura brasiliensis), de acordo com MACHADO et al. (2009a), apresentou topografia semelhante à registrada no lobo guará, ou seja, localizada entre L2 e L4, porém, com comprimento de $5 \mathrm{~cm}$, sendo este valor mais próximo ao registrado no Sauim. Já o lobo marinho (Arctocephalus australis), estudado por MACHADO et al. (2003), manifestou uma topografia do cone medular bastante curiosa quando comparada não só com os demais carnívoros aqui citados, bem como com o sauim, dispondo-se entre T5 e T7, com $4,4 \mathrm{~cm}$ de comprimento.

Confrontando com os achados em roedores selvagens, pode-se citar o cone medular da paca
(Agouti paca), o qual apresentou topografia próxima à do sauim, entre L5-7 e S1, com menor mensuração, registrando-se 3,39cm de comprimento (SCAVONE et al. 2007), porém, não menor que a registrada no ratão-do-banhado (Myocastor coypus), $2 \mathrm{~cm}$, localizado também entre L3 e L5 (MACHADO et al. 2009 b), assim como no lobo guará (MACHADO et al., 2002).

Por fim, e não menos importante, ainda em decorrência da escassa literatura sobre esse tema em primatas não-humanos, pode-se citar a semelhança topográfica do cone medular de dois Xenarthra, sendo um deles a preguiça comum (Bradypus variegatus), localizado entre L3 e S1, quando comparado com o sauim, entretanto, com apenas 50\% do comprimento, ou seja, registrando-se 2,7cm (LIMA et al. 2011); e o tamanduá-mirim (Tamandua tetradactyla), que não apresentou nenhuma semelhança com os achados no sauim, tanto no quesito mensuração, quanto topograficamente, pois localizou-se entre L1 e L5-6 com 6,5 a $7 \mathrm{~cm}$ de comprimento (MACHADO E SANTOS, 2008).

\section{CONCLUSÃO}

Mediante a topografia do cone medular do sauim (S. midas), pode-se sugerir uma abordagem segura para infusão de anestesia epidural nesssa espécie, entre os espaços interarqueados da região lombosacral, dispostos caudalmente a partir de L4 até S2.

\section{COMITÊ DE ÉTICA E BIOSSEGURANÇA}

Autorização no 485/2009, 522/2009.

\section{REFERÊNCIAS}

ARAÚJO, A. et al. Body weight of wild and captive common marmosets (Callithrix jacchus). International Journal of Primatology, Durham, v.21, n.2, p.317-324, 2000. Disponível em: <http://www.springerlink.com/content/p43407kr43310148/ fulltext.pdf $>$. Acesso em: 09 nov. 2011.

AURICHIO, P. Primatas do Brasil. São Paulo: Terra Brasilis, 1995. $168 \mathrm{p}$.

BRÍGIDA, S.S.S. et al. Topografia do cone medular da jaguatirica (Leopardus pardalis). Acta Veterinária Brasilica, Mossoró, v.4, n.1, p.51-54, 2010. Disponível em: <http://ojs.c3sl.ufpr.br/ojs2/ index.php/veterinary/article/viewFile/4031/3262>. Acesso em: 20 abr. 2010. doi: 10.1590/S0103-84782008000400014.

CARVALHO, S.F.M. et al. Topografia do cone medular em um gato-mourisco, Herpailurus yagouaroundi (Severtow, 1858) (FELIDAE). Archives of Veterinary Science, Curitiba, v.8, n.2, p.35-38, 2003. Disponível em: <http://ojs.c3sl.ufpr.br/ojs2/index. php/veterinary/article/viewFile/4031/3262>. Acesso em: 06 nov. 2011. doi: ISSN: 1517-784X. 
DYCE, K.M. et al. Tratado de anatomia veterinária. 3.ed. Rio de Janeiro: Guanabara Koogan, 2004. 813p.

FANTONI, D.T.; CORTOPASSI, S.R.G. Anestesia em cães e gatos. 2.ed. São Paulo: Roca, Brasil, 2002. 389p.

GREGORES, G.B. et al. Topografia do cone medular do quati (Nasua nasua Linnaeus, 1766). Biotemas, Florianópolis, v.23, n.2, p.173-176, 2010. Disponível em: <http://www.biotemas.ufsc. br/volumes/pdf/volume232/173a176Final.pdf $>$. Acesso em: 06 nov. 2011. doi: ISSN 0103 - 1643.

HERSHKOVITZ, P. Living new world monkeys (Platyrrhini) with an introduction to primates. Chicago and London: University of Chicago, 1977. V 1, 1132p.

HILBERY, A.D.R. Manual de anestesia de los pequeños animales. Zaragoza: Acribia, 1992. 154p.

HOPKINS, G.S. The correlation of anatomy and epidural anesthesia in domestic mammals. Cornell Veterinarian, Ithaca, NY, v.25, p.263-270, 1935

INTERNATIONAL COMMITTEE ON VETERINARY GROSS ANATOMICAL NOMENCLATURE. Nomina anatomica veterinária. 5.ed. Hannover: Editorial Committee, 2005. 165p.

LIMA, A.R. et al. Topografia do cone medular da preguiça Pesquisa Veterinária Brasileira, v.31, p.627-630, 2011. Disponível em: <http://www.pvb.com.br/pdf_artigos/31-072011_17-08Vet\%201011_2218\%20MF.pdf>. Acesso em: 06 nov. 2011. doi: ISSN 0100-736X.

MACHADO, G.V. et al. Topografia do cone medular no loboguará (Chrysocyon brachyurus Illiger, 1815). Revista Brasileira de Ciência Veterinária, Niterói, v.9, n.2, p.107-109, 2002.

MACHADO, G.V. et al. Topografia do cone medular no ratãodo-banhado (Myocastor coypus Molina, 1782 - Rodentia: Mammalia). Biotemas, Florianópolis, v.22, n.2, p.117-120, 2009b. Disponível em: <http://www.biotemas.ufsc.br/volumes/ pdf/volume222/117a120.pdf $>$. Acesso em: 06 nov. 2011. doi: ISSN $0103-1643$.

MACHADO, G.V. et al. Topografia do cone medular no lobomarinho (Arctocephalus australis Zimmermann, 1803). Arquivos de Ciências Veterinárias e Zoologia da UNIPAR, Umuarama, v.6, n.1, p.11-14, 2003. Disponível em: <http://revistas.unipar.br/ veterinaria/article/viewFile/787/687>. Acesso em: 06 nov. 2011. doi: ISSN 1415-8167.
MACHADO, G.V. et al. Esqueletopia do cone medular em Callithrix jacchus jacchus. In: CONGRESSO BRASILEIRO DE MEDICINA VETERINÁRIA, 22., 1992, Curitiba, PR. Anais... Curitiba: SBMV, 1992. p.56.

MACHADO, G.V. et al. Topografia do cone medular na ariranha (Pteronura brasiliensis Zimmermann, 1780). Ciência Animal Brasileira, Goiânia, v.10, n.1, p.301-305, 2009a. Disponível em: $<$ http://www.revistas.ufg.br/index.php/vet/article/view/4093/4598>. Acesso em: 06 nov. 2011. doi: ISSN 1089-6891.

MACHADO G.V.; SANTOS B.S. Topografia do cone medular no tamanduá-mirim (Tamandua tetradactyla Linnaeus, 1758) (Xenarthra: Myrmecophagidae). Archives of Veterinary Science, Curitiba, v.13, n.3, p.172-175, 2008. Disponível em: $<$ http://ojs. c3sl.ufpr.br/ojs2/index.php/veterinary/article/view/11666/9820>. Acesso em: 06 nov. 2011. doi: ISSN 1517 - 784X

NAGAMACHI, C.Y. et al. Cytogenetic study os Saguinus midas midas (Callithricidae, Primata) from Jari, brasilian amazon region. Comparison with the karyotype of Saguinus midas niger. Revista Brasileira de Genética, v.13, n.1, p.89-96, 1990. Disponível em: <http://web2.sbg.org.br/gmb/edicoesanteriores/v13n1/pdf/ a08v13n1.pdf $>$. Acesso em: 06 nov. 2011. doi: ISSN 0100-8455.

PONTES, A.R.M. et al. Fragmentation causes rarity in common marmosets in the Atlantic Forest of North-eastern Brazil. Biodiversity and Conservation, v.16, n.4, p.1175-1182, 2007. Disponível em: <http://www.mendeley.com/research/ fragmentation-causes-rarity-in-common-marmosets-in-theatlantic-forest-of-northeastern-brazil/>. Acesso em: 09 nov. 2011. ISSN: 09603115. doi: 10.1007/s10531-006-9099-5.

SCAVONE A.R.F. et al. Topografia do cone medular da paca (Agouti paca, Linnaeus - 1766). Brazilian Journal of Veterinary Research and Animal Science, São Paulo, v.44, p.53-57, 2007. Disponível em: $<$ http://www.fumvet.com.br/periodico/44/sup_5357.pdf>. Acesso em: 06 nov. 2011. doi: ISSN 1413-9596.

RAO, G.S. Anatomic studies on ovine spinal cord. Anatomischer Anzeiger, Erlangen, v.71, p.261-264, 1990.

RAO, G.S. et al. Anatomical studies on the spinal cord segments of the impala (Alpycerus melanus). Zentralblatt fur Veterinarmedizin, Reihe C, Anatomia, Histologia, Embryologia, v.22, p.273-278, 1993.

WRIGHT, E.M.; BUSH, D.E. The reproductive cycle of the Capuchin (Cebus paella). Laboratory Animal Science, v.5, p.651-654, 1977.

Ciência Rural, v.43, n.6, jun, 2013. 